\title{
The changes of subtypes in pediatric diabetes and their clinical and laboratory characteristics over the last 20 years
}

\author{
Eun Byul Kwon, MD', \\ Hae Sang Lee, MD, PhD², \\ Young Seok Shim, $\mathrm{MD}^{3}$, \\ Hwal Rim Jeong, $\mathrm{MD}^{4}$, \\ Jin Soon Hwang, MD, $\mathrm{PhD}^{2}$ \\ ${ }^{1}$ Department of Pediatrics, CHA \\ Bundang Medical Center, CHA \\ University, Seongnam, ${ }^{2}$ Department \\ of Pediatrics, Ajou University Hospital \\ Ajou University School of Medicine, \\ Suwon, ${ }^{3}$ Department of Pediatrics, \\ Hallym University Medical Center, \\ Hallym University College of Medicine, \\ Seoul, ${ }^{4}$ Department of Pediatrics, \\ Masan medical center, Changwon, \\ Korea
}

Purpose: We studied the changes in subtypes of diabetes mellitus (DM) in children and evaluated the characteristics of each group over the past 20 years. In addition, we also examined the correlation between the glycated hemoglobin ( $\mathrm{HbA1c}$ ) values at the time of diagnosis and lipid profiles.

Methods: The patients were divided into 2 groups: there were a total of 190 patients under 20 years of age firstly diagnosed with DM in Ajou University Hospital. The patients in groups I and II were diagnosed from September 1995 to December 2004 and from January 2005 to April 2014, respectively.

Results: The characteristics were compared between the 2 groups of patients. The result showed an increase in percentage of type 2 diabetes and maturity onset diabetes of the young (MODY) patients between the 2 groups. HbA1c and total cholesterol level had statistical significances to explain increasing the lowdensity lipoprotein cholesterol level among age, $\mathrm{HbA} 1 \mathrm{c}$, total cholesterol level, and $z$-scores of weight and body mass index (BMI) in type 2 diabetes. R-square was 0.074 . However, $z$-score of BMI and total cholesterol level, not HbA1c, had statistical significances in type 1 diabetic patients. R-square was 0.323 .

Conclusion: The increase in the proportions of both type 2 diabetes and MODY in the last 10 years needed to be reminded when diagnosing the subtypes of DM, and the dyslipidemia should be attended more as a common problem of pediatric diabetic patients.

Keywords: Type 2 diabetes mellitus, Child, Adolescent

\section{Introduction}

Type 2 diabetes in Korea and other Asian countries has gradually increased over the last 30 years ${ }^{1,2)}$, which can occur in children as well as adults. A previous study showed that type 2 diabetes in children was found before 1990, but the incidence of pediatric type 2 diabetes are $5.3 \%$ ( 1 of 19) and 21.0\% (8 of 38) in the years 1990 and 2000 in Korea, respectively ${ }^{3)}$. However, there were few studies after the year 2000. Therefore, researching subtypes of diabetes mellitus (DM) have a meaning itself and can be helpful to diagnose, establish treatment plans, and investigations.

Moreover, lipid profiles of patients are ascertained in this study. Dyslipidemia is an emerging problem in Korea and Asian countries as one of the important modifiable risk factors for cardiovascular disease, in all children including diabetic patients ${ }^{4,5)}$. Dyslipidemia-insulin resistance-hyperinsulinemia vicious cycle can cause inflammation, endoplasmic reticulum stress and lipotoxicity in diabetic patients and affect their progress ${ }^{6,7)}$. Therefore, dyslipidemia should be attended more by clinicians, but the concern and/or eager to the treatment are less in the children than the adults.

\footnotetext{
Received: 4 February, 2016

Revised: 10 March, 2016

Address for correspondence:

Jin Soon Hwang, MD, PhD

Department of Pediatrics, Ajou University Hospital, Ajou University School of Medicine, 164 World cupro, Yeongtong-gu, Suwon 16499, Korea

Tel: +82-31-219-5166,

Fax: +82-31-219-5169,

E-mail: pedhwang@ajou.ac.kr
} 
In this study, therefore, the changes in subtypes of diabetes and their clinical and laboratory characteristics were reviewed from the viewpoint of the changes in subtypes of pediatric diabetes patients and their lipid profiles correlated with glycemic control.

\section{Materials and methods}

This was a retrospective study for ascertaining whether subtype of diabetes were really changed using the chart review and taking a look at the relationship between glucose and cholesterol level. We included all pediatric patients (under the age of 20) who firstly diagnosed diabetes during the years from September 1995 to April 2014 in Ajou University Hospital. The population was comprised of 190 children. They were divided into groups I and II, diagnosed during the years from September 1995 to December 2004 and from January 2005 to April 2014, respectively. Each of the 2 different groups was subdivided into 3 different subgroups: type 1 diabetes, type 2 diabetes, and maturity onset diabetes of the young (MODY). Patient information such as age at diagnosis, gender, height, weight, body mass index (BMI), family history of diabetes, antiislet cell autoantibodies, serum cholesterol and triglycerides, high-density lipoprotein cholesterol (HDL-C), and low-density lipoprotein cholesterol (LDL-C) was collected from chartrecords. Blood chemistry test was done in the morning with fasting state in the second day of admission.

Diagnostic criteria of type 1 diabetes were as follows ${ }^{3)}$ : (1) patients with ketoacidosis, (2) fasting serum C-peptide level below $0.6 \mathrm{ng} / \mathrm{mL}$ and (3) positive anti-islet cell antibodies or weight under 50th percentile as well as glycemic control impossible without insulin injection although the patients had fasting serum C-peptide level over $0.6 \mathrm{ng} / \mathrm{mL}$.

Diagnostic criteria of MODY were identified in the patients having a family history with onset under 25 years of age through 3 and more consecutive generations, having nonautoimmune type, and good response to sulfonylurea. Insulin autoantibody (IAA), antiglutamic acid decarboxylase antibody (anti-GAD $\mathrm{Ab})$ and islet cell antibody were measured among anti-islet cell autoantibodies. Positive response was defined as 0.9 units $/ \mathrm{mL}$ and higher anti-GAD Ab level or 7\% and higher IAA level.

Statistical analyses were conducted using IBM SPSS Statistics ver. 19.0 (IBM Co., Armonk, NY, USA) and P-values under 0.05 were reported as statistically significant. The comparison of continuous data and analysis of categorical variables were performed using Student $t$-test, and either chi-square test or Fisher exact test, respectively. Robust analysis of variance by Welch et al. was conducted for comparing among three different diabetic subtypes because homogeneity of variance between each subtype was not satisfied. Multiple regression analysis was also performed to estimate the magnitude of association between glycated hemoglobin (HbAlc) at the time of diagnosis and serum lipid level.

\section{Results}

There were 52 patients and 138 patients in groups I and II, respectively, and their basic characteristics are shown in Table 1. The values between the 2 groups such as age at diagnosis, gender, and height showed no statistically significant difference. Although weight and BMI were greater in group II than in group I, their z-scores showed no statistical significance. While $33 \%$ and $52.9 \%$ of patients in groups I and II had family history of DM, respectively, showing statistical significance $(P=0.01)$.

Fig. 1 shows the proportion of DM subtypes in the 2 different groups. Group I had $84.6 \%$ proportion of type 1 diabetes, $11.5 \%$ proportion of type 2 diabetes and 3.9\% proportion of MODY, whereas group II had $65.9 \%$ proportion of type 1 diabetes, $24.0 \%$ proportion of type 2 diabetes and $10.1 \%$ proportion of MODY. These results showed statistical significance $(P=0.04)$.

Table 2 shows the lipid levels in DM patients. There was no statistically significant difference except for triglyceride in the serum lipid levels over the last 20 years, which is consistent with the result of classification according to the subtype of DM. In multiple regression tests, HbAlc and total cholesterol level had statistical significances to explain the increase of the LDL-C level among age, $\mathrm{HbAlc}$, total cholesterol level, and $z$-scores of weight and BMI in type 2 diabetes. R-square was 0.074 .
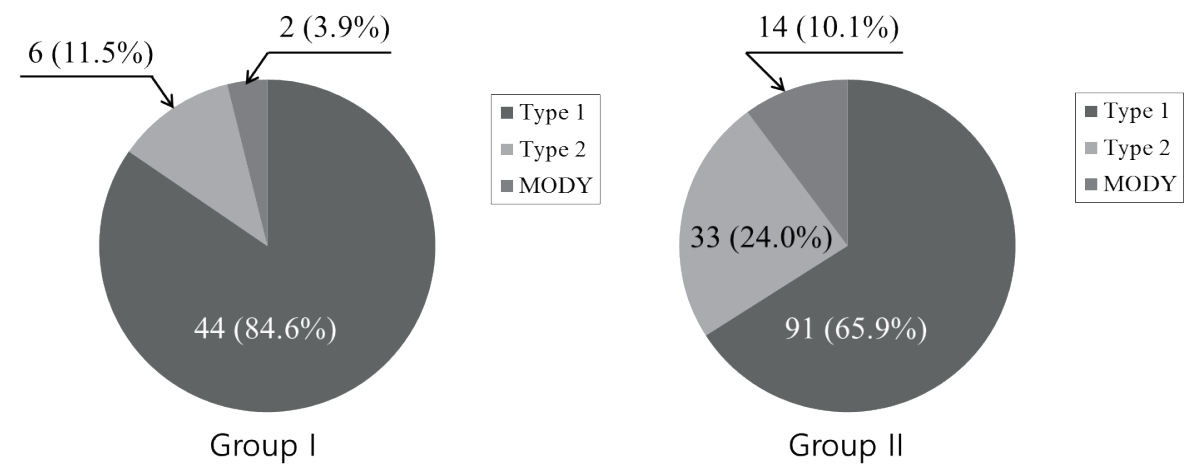

Fig. 1. Change of diabetes mellitus types with 2 different periods. Group I (diagnosed from 1995 to 2004); group II (diagnosed from 2005 to 2014); MODY, maturity onset diabetes of the young. 

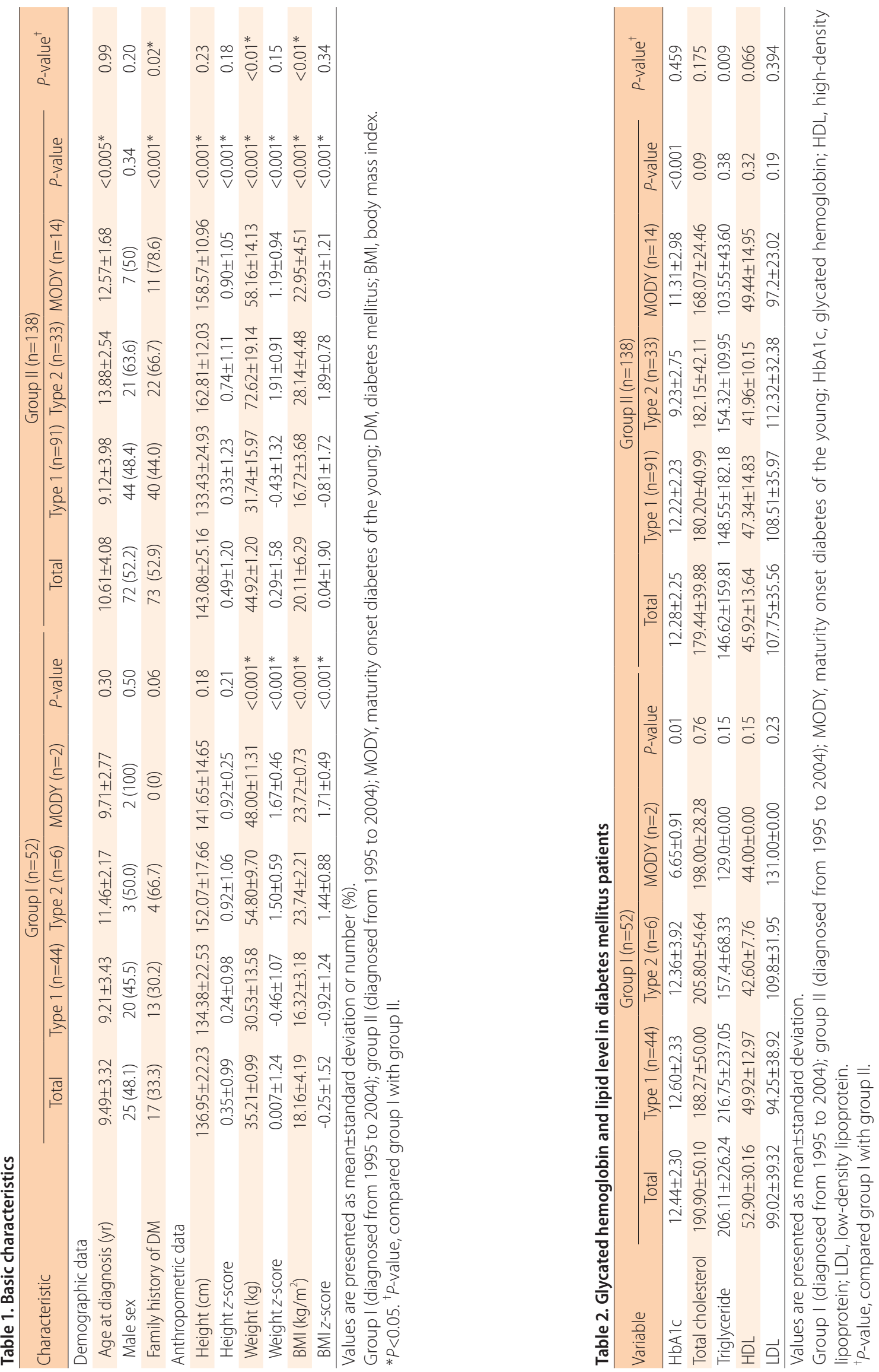
However, $z$-score of BMI and total cholesterol level, not HbAlc, had statistical significances in type 1 diabetic patients. R-square was 0.323

\section{Discussion}

Over the last 10 years, in this study, the proportion of type 2 diabetes in the patients diagnosed as having DM increased approximately twice from $11.5 \%$ in group I to $23.9 \%$ in group II, which suggested that it was related to an increase in the population of obese adolescents.

In a study by Bahk and Khang ${ }^{8}$, the proportion of obese male patients among total children and adolescents increased from $3.3 \%$ to $6.9 \%$ over different periods from 1998 to $2010-2012$, while the proportion of obese female patients among total children and adolescents increased from $4.7 \%$ to $7.7 \%$. The proportions of overweight and obese male children having acanthosis nigricans, meaning insulin resistance, were $11.4 \%$ and $45.2 \%$, respectively, while the proportions of overweight and obese female children having acanthosis nigricans were 5.9\% and $43.8 \%$, respectively ${ }^{9)}$.

In the previous study, it was noticeable that the proportion of central obesity, which was related to insulin resistance and metabolic syndrome, was higher among both children and adults in Asian countries compared to those with similar weight and BMI in Western countries ${ }^{10,11)}$.

This result was consistent with our practical study, which suggested that there was no statistically significant difference in $z$-score of weight and BMI between the 2 groups, while the number of patients diagnosed as having type 2 diabetes was remarkably increased over the last 10 years. Unfortunately, data about abdominal circumference and/or insulin resistance could not be obtained, which could have made our study more valuable.

In this study, age at diagnosis of type 2 diabetes was higher in group II (diagnosed during the period from 2005 to 2014). Type 2 diabetes was affected by puberty, which increased the insulin resistance and induced hyperinsulinemia. Both basal insulin reaction and stimulated insulin reaction increased after puberty. There were many cases suggesting that an increase in the growth hormone concentration induced insulin resistance during puberty, thereby leading to diagnosis of type 2 diabetes in midpuberty ${ }^{12,13)}$.

Generally, the type 2 diabetes patients have elevated triglycerides, low HDL-C cholesterol, and predominance of small-dense LDL-C particles, which might be related to the modulation of 3-hydroxy-3-methylglutaryl-coenzyme A-reductase. The dyslipidemia is based on inflammatory processes and on flooding of the body with energy rich substrates (glucose and/ or free fatty acids) which then results in hepatic and intestinal lipoprotein overproduction leading to hypertriglyceridemia (fasting and postprandial), which in turn stimulates the secretion of apolipoprotein $B(A p o B)$ and very LDL cholesterol (VLDL-C). In addition to high ApoB and VLDL-C, hyperinsulinemia is increased catabolism of HDL-C (resulting in low HDL-C) $)^{6,7,14,15)}$. So, poorly controlled type 2 diabetes diabetic patient have more severe dyslipidemia.

Although few data exist on the effects of dyslipidemia on patients with typel diabetes, some studies report that rates of dyslipidemia in the general pediatric population and in pediatric patients with well-controlled type 1 diabetes patients are similar. In contrast, in patients with type 2 diabetes, even when in good glycemic control, there are abnormalities in lipid levels, in other words, youth with type 1 diabetes also had significantly elevated Apo B levels and VLDL-C than nondiabetic youth, regardless of glycemic control $^{16-18)}$.

$\mathrm{HbAlc}$ reflects the concentration of serum glucose level over several weeks preceding the test. The positive correlation between $\mathrm{HbAlc}$ and cholesterol level in this study caused by hyperglycemic state which then result in hypertriglycemic and increased LDL-C state ${ }^{6,7,14-18)}$.

The results of multiple regression analysis for LDL-C match to existing theory. Although factors influencing the determination of LDL-C level are variable for both types 1 and 2 diabetic patients, BMI $z$-score is more important consideration in the LDL-C level of type 1 diabetes patients. Whereas LDL-C level of type 2 diabetes patients affected by $\mathrm{HbAlc}$ level.

Nevertheless, this was a retrospective study performed at only a single tertiary medical center, which may not be representative of the population. In this study, there were some limitations such as the change in the treatment could not be analyzed during follow-up. However, the number of diabetic patients was large enough to study it as a single disease entity.

In conclusion, there was an increase in the proportions of both type 2 diabetes and clinically defined MODY in the patients diagnosed as having DM over the last 10 years. Moreover, the increase in the HbAlc at the diagnosis was related to that in total cholesterol and LDL-C levels in type 2 diabetes. Therefore, further study is needed for glycemic control of pediatric DM patients having dyslipidemia.

\section{Conflict of interest}

No potential conflict of interest relevant to this article was reported.

\section{References}

1. Yoon KH, Lee JH, Kim JW, Cho JH, Choi YH, Ko SH, et al. Epidemic obesity and type 2 diabetes in Asia. Lancet 2006;368:1681-8.

2. Ma RC, Chan JC. Type 2 diabetes in East Asians: similarities and differences with populations in Europe and the United States. Ann N Y Acad Sci 2013;1281:64-91.

3. Park J, Oh J, Yu J. Autoantibody positivity and clinical characteristics of diabetes mellitus in childhood. J Korean Soc Pediatr Endocrinol 2011;16:119-27.

4. Yang S, Hwang JS, Park HK, Lee HS, Kim HS, Kim EY, et al. Serum lipid concentrations, prevalence of dyslipidemia, 
and percentage eligible for pharmacological treatment of Korean children and adolescents; data from the Korea National Health and Nutrition Examination Survey IV (2007-2009). PLoS One 2012;7:e49253.

5. Lim S, Jang HC, Park KS, Cho SI, Lee MG, Joung H, et al. Changes in metabolic syndrome in American and Korean youth, 1997-2008. Pediatrics 2013;131:e214-22.

6. Zhu XW, Deng FY, Lei SF. Meta-analysis of Atherogenic Index of Plasma and other lipid parameters in relation to risk of type 2 diabetes mellitus. Prim Care Diabetes 2015;9:60-7.

7. Li N, Fu J, Koonen DP, Kuivenhoven JA, Snieder H, Hofker MH. Are hypertriglyceridemia and low HDL causal factors in the development of insulin resistance? Atherosclerosis 2014;233:130-8.

8. Bahk J, Khang YH. Trends in measures of childhood obesity in Korea from 1998 to 2012. J Epidemiol 2016;26:199-207.

9. Chang Y, Woo HY, Sung E, Kim CH, Kang H, Ju YS, et al. Prevalence of acanthosis nigricans in relation to anthropometric measures: community-based crosssectional study in Korean pre-adolescent school children. Pediatr Int 2008;50:667-73.

10. Lim H, Xue H, Wang Y. Association between obesity and metabolic co-morbidities among children and adolescents in South Korea based on national data. BMC Public Health 2014;14:279.

11. Kim HM, Park J, Kim HS, Kim DH, Park SH. Obesity and cardiovascular risk factors in Korean children and adolescents aged 10-18 years from the Korean National
Health and Nutrition Examination Survey, 1998 and 2001. Am J Epidemiol 2006;164:787-93.

12. Reinehr T. Type 2 diabetes mellitus in children and adolescents. World J Diabetes 2013;4:270-81.

13. Yi KH, Hwang JS, Kim EY, Lee SH, Kim DH, Lim JS. Prevalence of insulin resistance and cardiometabolic risk in Korean children and adolescents: a population-based study. Diabetes Res Clin Pract 2014;103:106-13.

14. Parhofer KG. Interaction between glucose and lipid metabolism: more than diabetic dyslipidemia. Diabetes Metab J 2015;39:353-62.

15. Leon BM, Maddox TM. Diabetes and cardiovascular disease: Epidemiology, biological mechanisms, treatment recommendations and future research. World J Diabetes 2015;6:1246-58.

16. Katz M, Giani E, Laffel L. Challenges and opportunities in the management of cardiovascular risk factors in youth with type 1 diabetes: Lifestyle and Beyond. Curr Diab Rep 2015;15:119.

17. Guy J, Ogden L, Wadwa RP, Hamman RF, Mayer-Davis EJ, Liese AD, et al. Lipid and lipoprotein profiles in youth with and without type 1 diabetes: the SEARCH for Diabetes in Youth case-control study. Diabetes Care 2009;32:416-20.

18. Feingold KR, Grunfeld C. Diabetes and dyslipidemia. In: De Groot LJ, Beck-Peccoz P, Chrousos G, Dungan K, Grossman A, Hershman JM, et al., editors. Endotext [Internet]. South Dartmouth (MA): MDText.com, Inc.; c2000-2016 [cited 2016 Mar 8]. Available from: www.endotext.org. 